\title{
Observations of Giant Pulses from Pulsar PSR B1937+21
}

\author{
S. E. Thorsett ${ }^{1}$, J. A. Shrauner ${ }^{1}$, I. Cognard ${ }^{1,2}$, J. H. Taylor ${ }^{1}$ \\ ${ }^{1}$ Physics Department, Princeton University, Box 708, Princeton NJ \\ 08544 USA \\ ${ }^{2}$ Laboratoire de Physique et Chimie de l'Environnement, Orleans, France
}

\begin{abstract}
We discuss observations of giant pulses from the millisecond pulsar PSR B1937+21 at $430 \mathrm{MHz}$, with a baseband sampling system at Arecibo (Shrauner et al., these proceedings) and coherent dedispersion techniques. About one pulse or interpulse per hundred thousand is stronger than 100 times the mean flux density, and the giant pulse strengths follow a power law distribution somewhat shallower than that of the Crab giant pulses. The individual giant pulses appear consistent with impulses shorter than a few microseconds, convolved with an exponential due to interstellar scattering. They are systematically delayed with respect to the average emission, and many are nearly $100 \%$ circularly polarized.
\end{abstract}

Since the IAU Colloquium, the work presented in this talk has appeared in the refereed literature (Cognard et al. 1996).

\section{References}

Cognard, I., Shrauner, J. A., Taylor, J. H., Thorsett, S. E., 1996, ApJ, 457, L81. 